\title{
MATHEMATICAL MODELS IN ECOCHEMISTRY
}

\author{
J. ROBINSON
}

Tunstall Laboratory, Shell Research Ltd, Sittingbourne, Kent, UK

\begin{abstract}
General concepts concerning models, their logical and analogical status, are briefly outlined. The use of compartmental models to simulate the kinetics of compounds in the environment is illustrated by a discussion of carbon dioxide in the atmosphere and oceans and its uptake by plants. The application of compartmental models to the kinetics of organochlorine compounds in vertebrates and in ecosystems is reviewed and the simplifying assumptions are made explicit. It appears from the evidence available, and assuming the compartmental models are valid simulations of ecosystems, that there may be significant mechanisms of removal of organochlorine insecticides from the biosphere, possibly by chemical degradation in the troposphere or stratosphere; these mechanisms require detailed investigation to assess their importance.
\end{abstract}

\section{INTRODUCTION}

The study of the behaviour of chemicals in ecosystems, and in the biosphere, has progressed substantially during the past 20 years, not only in the collection of reliable information on the occurrence and kinetics of chemicals in the environment, but also in the development of models that simulate the changes in time and space of the concentration of a chemical. The major advances in the development of models in ecochemistry have come from the study of substances such as carbon dioxide, carbon monoxide, sulphur dioxide, compounds of lead and mercury, and fallout of radioactive isotopes, but significant contributions have also come from investigations of the contamination of ecosystems by organochlorine compounds.

As with other studies of the oikos, ecochemistry is a combination of disparate scientific disciplines: sampling theory and statistical inference; meteorology, geochemistry, photochemistry and analytical chemistry; ecology and population dynamics; to name but a few. There are inevitable differences of approach, and these have been the source of strong disagreements between scientists of different disciplines. These divergences of opinion have been distorted and amplified in their transposition into television programmes, articles in the popular press, and books written for nonscientists.

A chemical is released into the enviroument: what predictions, if any, can we make about the fate of the chemical? What are the rates of transfer from, say, the soil to the atmosphere and vice versa? What are the concentrations at times $t_{1}$ and $t_{2}$, after the release began, in the eggs of a particular species of 
bird? What is the relationship between the addition of tetra-alkyl lead compounds to petrol and the concentration of lead in arctic snow? What happens when the release of the chemical is terminated? These are some of the many questions that we may ask ourselves. They have an obvious relevance to the assessment of the biological impact of a compound now or in the future if use of it is continued. The answers we give to these questions are to a large extent empirical, since we do not have sufficient knowledge to predict a priori what will happen. Many of the answers will be in the form of statistical inferences, since the environmental data will consist of stochastic variables. Further, if we wish to predict what will happen in the future rather than infer, in a synoptic manner, what occurred in the past or near present, then significant correlations between stochastic variables will rarely be a satisfactory basis for such predictions, since they will merely be inductive extrapolations. One way of improving the predictive power of our inferential procedures is by means of a theory which has been developed, possibly in the context of data of a different type from that under consideration, and which has withstood rigorous attempts to falsify it. If this theory can be expressed in the form of a mathematical model, then quantitative inferences may be drawn from a particular set of empirical observations using the model as a means of explicating the information in the observations.

\section{MODELS}

In broad terms a model is an image or analogue that simulates reality in some respect. There are many types of models, and failure to discriminate between them may have contributed to some of the controversies that have occurred in the history of science, and to some that are currently occurring about the impact of chemicals upon ecosystems. In logic, for example, the term 'model' is used with two distinct meanings. There are formal sentential models in which a set of sentences can be matched according to some rule with the sentences in which a theory is expressed; and there are iconic models in which some imagined thing or process behaves in a similar manner to some real thing or process. Iconic models are of great importance in science, since they are used as a means of establishing hypothetical causal mechanisms, or, if one adopts a more positivistic approach, functional relationships that are more efficient predictors than inductive inferences. There are several other ways of classifying models (see, for example, refs. 1 and 2), but the model of particular relevance to this paper is an iconic model expressed in mathematical terms, namely the compartmental model.

Some further general comments, on the conceptual schemes that we call models, are necessary. The relation between the model and the thing modelled is usually one of analogy, and there are two kinds of analogy present in models in science. Firstly, formal analogy based on an isomorphism derived from the same formal axiomatic and deductive relationships that connect individuals and predicates in both the system and the model. Secondly, material analogy in which the replica may have material similarities. In compartmental models the relationship of analogy is of the formal type. The relation of analogy, whether formal or material, implies differences as well as similarities, and we may denote the similarities as 'positive analogy' 
and the differences as 'negative analogy'. There are formal mathematical models in which no element of a replica is involved; their interpretation is in terms of concepts such as probability or geometry. Thus, a probabalistic (stochastic) model of population dynamics contains the axioms of probability theory together with an interpretation of all or some of the theory into empirical observations. Compartmental models have been used in biochemistry, toxicology and ecology, and a discussion of this type of model is warranted.

\section{COMPARTMENTAL MODELS}

A compartment is an abstraction: it is defined, in its broadest sense, as a set of elements. Various types of compartments can be delineated according to the defining property of the set, a defining property which is decided according to the purpose for which the compartment is to be used. So far as ecochemistry is concerned, there are two major types of compartment with separate but interrelated defining properties. In one case we are considering an organism in itself and the distribution and disposal of a chemical in that system; in the other case we consider organisms in relation to one another and their habitat. The first type of compartment, which has been very useful in biochemistry and toxicology, is defined as a set of elements in which the chemical potentials of a given chemical are equal at all times; any change of the chemical potential $\left(\mu_{i}^{\alpha}\right)$ of the $i$ th chemical in an $\alpha$-element is reflected instantaneously by an equal change in all the other elements of the compartment. It is further stipulated that the direction of movement of the $i$ th chemical into or out of an element is dependent on the difference between the $\mu_{i}^{\alpha}$ and the chemical potential of the $i$ th chemical in elements adjacent to it $\left(\mu_{i}^{\beta}\right)$. The work done in transferring $\mathrm{d} n$ molecules of the $i$ th chemical is thus $\left(\mu_{i}^{\alpha}-\mu_{i}^{\beta}\right) \mathrm{d} n$; if the change is spontaneous, then $\mu_{i}^{\alpha}>\mu_{i}^{\beta}$, and the transfer will continue until $\mu_{i}^{\alpha}=\mu_{i}^{\beta}$. Chemical potential can be defined, of course, as a function of changes in Helmholtz free energy or Gibbs free energy of the systems.

The activity $\left(a_{i}\right)$ of the $i$ th chemical in an element is an exponential function of its chemical potential:

$$
\mu_{i}^{\alpha}-\mu_{i}^{\alpha 0}=R T \ln a_{i}
$$

where $\mu_{i}^{\alpha 0}$ is an arbitrary constant for a given solute. If the elements are part of an open system (as is the case with a living organism) then the addition of an amount $\mathrm{d} n_{i}$ of the $i$ th solute to the system produces a change in the chemical potential:

$$
\mu_{i}=(\mathrm{d} G-V d P+S \mathrm{~d} T) / \mathrm{d} n_{i}
$$

and if several solutes are added simultaneously:

$$
\Sigma \mu_{i}=(\mathrm{d} G-V d P+S \mathrm{~d} T) / \Sigma \mathrm{d} n_{i}
$$

Finally, it is stipulated that the amount of the $i$ th solute undergoing chemical change at a given instant in an element is related to the activity of the solute at that instant. The concentrations of many solutes of ecological interest in the biophases of organisms are usually quite low (e.g. concentra- 
tion of organochlorine insectides in human adipose tissue is of the order of $10^{-6}$ molal; the concentrations in other macrobiophases are of the order $10^{-7}-10^{-8}$ molal). Such dilute solutions can probably be regarded as deviating insignificantly from ideal behaviour and the rate of reaction is consequently related to the concentration.

The second type of compartmental mode, in which we consider the individual organism as a member of the biotic community, is based upon the concept of a trophic level. A trophic level is defined as the set of organisms whose food is derived by the same number of steps from plants. All plants, therefore, belong to the same trophic level, usually called the first trophic level; all herbivores belong to trophic level two, primary carnivores to trophic level three, etc. The tropic classification is one of function (type of food) and not of species as such. Many species appear to be food generalists, and eat different types of food according to their availability. The availability of various types of food may vary from season to season and also over the range in which a particular species exists. Stenophagic organisms, with narrow preferences of food, seem to be much less common than euryphagic organisms (non-specialists or generalists). The biotic community may therefore be regarded as a collection of partially intersecting sets defined as trophic levels, since the members of a given species may occupy one or more trophic levels, either simultaneously or during different seasons. If one considers a particular biophase (e.g. adipose tissue, or plasma proteins), the chemical potential of a particular solute in this biophase in individual organisms may be very different (i.e. the concentrations of the solute may be very different), but, unlike the biophases within an organism, there is no mechanism of reversible exchange of molecules of a chemical in order to achieve equality of chemical potential. There are two cases to consider. The first is that of organisms in the same trophic level; by definition these organisms do not normally prey upon one another and thus molecules of a solute cannot be exchanged reversibly between organisms. The amount of a particular chemical in a particular biophase of members of a trophic level is dependent upon the amounts ingested per unit time by each individual and the duration of the ingestion (see below). In this case we may have significant correlations between residues in organisms, but there is no causal relationship. The second case is that of organisms in successive trophic levels; by definition the organisms in the $n$th trophic level eat those in the $(n-1)$ th trophic level. There is no mechanism of reversible exchange of a chemical between organisms, but there is a direct dependence in this case of the amount of the chemical in the $j$ th biophase of an organism in the $n$th trophic level upon the concentration of the chemical in the food of members of this trophic level. This concentration is, of course, that in organisms, or parts of organisms of the $(n-1)$ th trophic level. In this case we have a direct causal relationship between trophic levels, but the relationship is not reversible, neither is it a transitive causal relationship, although there may be significant transitive correlations.

So far we have considered only one mode of entry of a chemical into an organism, namely ingestion; this mode involves the transfer of a chemical across the membranes of the gastrointestinal tract to the blood and its subsequent distribution into the other tissues of the body. There are two other 
possible major routes of entry, namely by inhalation and by percutaneous absorption. Percutaneous absorption is not a major adventitious route of entry of most chemicals into the body, although it is important in certain sub-populations such as workmen with an occupational exposure to chemicals. Absorption through the respiratory tract is probably of minor importance for many chemicals, except, again, in the case of workmen with occupational exposure, or animals in the vicinity of the immediate application of pesticides, for example.

Although residues in the air are probably of minor significance as a source of exposure of animals to many chemicals, their occurrence in the atmosphere may provide a pathway for their transfer over large distances; further, in the case of certain chemicals such as carbon monoxide, methane, nitrous oxide and, possibly, low molecular weight fluorinated compounds (such as the Freons), the atmosphere is a major pathway for their dispersion in the biosphere. In addition to these compounds, some of which are mainly or even solely of anthropogenic origin, the atmosphere is a significant component in the transport of compounds such as carbon dioxide which, until recently, were predominantly of natural origin.

\section{COMPARTMENTAL MODEL FOR CARBON DIOXIDE}

The kinetics of carbon dioxide in the biosphere has been the subject of intensive research, and consequently the models that have been developed are considerably more sophisticated than those for most compounds. The models have become of increasing complexity as the number of compartments has increased from two ${ }^{3}$ (atmosphere and sea), to three ${ }^{4}$ (atmosphere, upper mixed layer, and deep ocean), to five (in which the two additional compartments are long-lived and short-lived biota); the division of the ocean into more than three reservoirs has also been investigated. As we are concerned more with basic principles of the use of models, and not with the more sophisticated ones based on five compartments even including modifications to allow for isotopic fractionation factors and transport of particulate matter, only the three-reservoir model will be considered in this paper.

In the pre-industrial era, we assume that man's activities had had little effect upon the amounts of carbon dioxide in the three compartments (this assumption is not strictly correct, since the burning of wood by man without adequate re-afforestation must have changed the atmospheric concentration of carbon dioxide from that prevailing prior to the Pleistocene epoch, but the relatively rapid and severe climatic changes during that epoch probably also affected the concentration of carbon dioxide in the atmosphere, and it would be difficult to isolate anthropogenic changes from the natural ones; in any case, the rate of generation of carbon dioxide by man's activities prior to the industrial period would be small compared with that during the last 50 years or so). It is also plausible to assume that the biosphere was in a steady state prior to the industrial period; i.e. the transfer of carbon dioxide into any compartment was balanced by the transfer of carbon dioxide from it. Further, the compartments are distinguished by their physical properties (gas or liquid phase, the latter being subdivided into water above the thermocline and water below it) and have the property that the transfer of 
carbon dioxide from one reservoir to another takes a relatively long time to reach equilibrium (say one year or more) compared with the mixing time within a compartment. Finally, we assume that the transfer of carbon dioxide from a reservoir is proportional to the total amount of carbon dioxide in that reservoir at that instant.

The steady state equations for a model of the pre-industrial period are:

$$
\begin{aligned}
& \mathrm{d} Q_{1} / \mathrm{d} t=-k_{12} Q_{1}+k_{21} Q_{2}=0 \\
& \mathrm{~d} Q_{2} / \mathrm{d} t=k_{12} Q_{1}-k_{21} Q_{2}-k_{23} Q_{2}+k_{32} Q_{3}=0 \\
& \mathrm{~d} Q_{3} / \mathrm{d} t=k_{23} Q_{2}-k_{32} Q_{3}=0
\end{aligned}
$$

where $Q_{1}$ is the quantity of carbon dioxide in the atmosphere, $Q_{2}$ the quantity in the upper mixed layer of the ocean (i.e. to a depth of about $100 \mathrm{~m}$ ) and $Q_{3}$ the quantity in the deep ocean; $k_{i j}$ is the transfer constant for the movement of carbon dioxide from the $i$ th to the $j$ th compartment.

We have a set of three simultaneous linear differential equations which can be solved, but it is convenient to consider the corresponding equations for a model of the industrial period, in which there is an input of carbon dioxide into the atmosphere as a result of man's increased utilization of fossil fuels (for simplicity we assume that this rate of input, $\dot{q}$, is constant):

$$
\begin{aligned}
& \mathrm{d} Q_{1} / \mathrm{d} t=\dot{q}-k_{12} Q_{1}+k_{21} Q_{2} \\
& \mathrm{~d} Q_{2} / \mathrm{d} t=k_{12} Q_{1}-k_{21} Q_{2}-k_{23} Q_{2}+k_{32} Q_{3} \\
& \mathrm{~d} Q_{3} / \mathrm{d} t=k_{23} Q_{2}-k_{32} Q_{3}
\end{aligned}
$$

This set of simultaneous linear differential equations is conveniently solved using the appropriate Laplace transform (i.e. by multiplying each term in equation 2 by $\exp (-s t)$, the general solution being:

$$
Q_{i}=A_{i} \mathrm{e}^{\lambda_{1} t}+B_{i} \mathrm{e}^{\lambda_{2} t}+C_{i} \mathrm{e}^{\lambda_{3} t}+D_{i}
$$

where $A_{i}, B_{i}$ and $C_{i}$ are integration constants for the $i$ th reservoir, $D_{i}$ is dependent upon the time-dependent character of $\dot{q}(t)$, and $\lambda_{1}, \lambda_{2}$ and $\lambda_{3}$ are functions of $k_{12}, k_{21}$, and $k_{23}$ and $k_{32}$. This model may be extended to include transfers of carbon dioxide between the atmosphere and terrestrial plants; if the quantity of equivalent- $-\mathrm{CO}_{2}$ in the plants is denoted by $Q_{4}$, and the transfer coefficient, atmosphere to plant and plant to atmosphere by $k_{14}$ and $k_{41}$, respectively, then a set of four linear differential equations analogous to those in equation 2 may be derived and integrated. A discussion of the method of solving the equations of the above and more complex models, together with comparisons of the predicted and observed values of atmospheric carbon dioxide is given by Keeling 5 .

The development of a compartmental model for carbon dioxide illustrates a number of general principles, but there are some particular aspects of the behaviour of carbon dioxide that must be noted before we attempt to generalize the above type of model for all ecochemicals. Thus, carbon dioxide is relatively soluble in water, and undergoes chemical reaction to give the bicarbonate ion, thereby reducing the partial pressure of carbon dioxide in the upper mixed layer of the ocean. Further, it has the specific property 
of being converted in plants by photosynthesis into carbohydrates. These pathways of removal are insignificant for many compounds.

\section{COMPARTMENTAL MODELS FOR SOME ORGANOCHLORINE COMPOUNDS}

In recent years a number of organochlorine compounds, such as DDT, dieldrin and the polychlorinated biphenyls, have become the focus of considerable attention and controversy. Residues of organochlorine compounds have been found in many types of samples, including air, rainwater, human tissues, fish, and the tissues and eggs of a large number of avian species. These compounds have relatively low vapour pressures, very slight solubility in water (less than 0.1 p.p.m. in some cases) and are quite stable in many components of the biosphere. Tentative suggestions about possible models to simulate the behaviour of organochlorine compounds in organisms and ecosystems, based upon the mamillary-type compartmental models for organisms, and one utilizing the trophic level concept for ecosystems, were made by Robinson ${ }^{6}$. A more explicitly developed model for DDT was proposed by Harrison et al. ${ }^{7}$. The latter authors simplified the mathematical analysis of their model for DDT by assuming that organisms in all consumer levels metabolize DDT at an insignificant rate. It has been pointed out that this assumption is incorrect for $p p^{\prime}$-DDT and other organochlorine insecticides such as dieldrin ${ }^{8}$. Nevertheless, the model proposed by Harrison et al., without this simplifying assumption, warrants detailed discussion.

Before discussing the kinetics of pesticides in ecosystems and in the biosphere, utilizing the trophic level concept, it is necessary to discuss first the kinetics of pesticides in organisms. The organisms studied in the majority of investigations of this type are vertebrates. It was proposed some years ago $^{6}$, on the basis of the rather meagre information published at that time, that the kinetics of organochlorine insecticides such as $p p^{\prime}$-DDT and dieldrin in vertebrates were consistent with the four following propositions:

(1) The concentration of an organochlorine insecticide in a particular tissue is a function of the daily intake:

$$
c_{\alpha i j}=f_{\alpha i j}(a)
$$

where $c_{\alpha i j}$ is the concentration of the $j$ th insecticide in the $i$ th tissue of an animal of species $\alpha$, and $a$ is the daily intake of that compound.

(2) The concentrations of the insecticide in different tissues are functionally related:

$$
c_{\alpha i j}=g_{\alpha i j k}\left(c_{\alpha j k}\right)
$$

where $c_{\alpha i j}$ and $c_{\alpha k j}$ are the concentrations of the compound in the $i$ th and $k$ th tissues.

(3) The concentration of the compound in a particular tissue is dependent on the duration of the ingestion of the insecticide:

$$
c_{\alpha i j}=h_{\alpha i j}(t)
$$

The function $h_{\alpha i j}(t)$ has a finite upper limit which is approached asymptotically 
as the duration $(t)$ of the ingestion is increased (unless, of course, the concentration in some target organ reaches an effect value and elicits a harmful or lethal response).

(4) When the ingestion of the compound is terminated, the concentrations on the tissues declines and the rates of decrease at a given time are proportional to the concentrations in the tissues at that time:

$$
\mathrm{d}\left(c_{\alpha i j}\right) / \mathrm{d} t=-i_{\alpha i j}\left(c_{\alpha i j}\right)
$$

In some cases a semilogarithmic relationship fits the data satisfactorily (corresponding to a pseudo-first-order type of elimination); in other cases more complex relationships exist. There are probably a variety of empirical relationships that give a satisfactory fit in these cases (e.g. a power function was suggested in the case of $p p^{\prime}$-DDT in the body-fat of steers, but a relationship of this type was not appropriate for dieldrin in the liver of rats).

It would perhaps be inappropriate to claim that information published since these propositions were suggested has confirmed them, but it appears that they have not been falsified (i.e. as judged by Popper's criteria for assessing the validity of a thesis ${ }^{9-11}$ ).

It has been pointed out that these empirical propositions are consistent with a compartmental model of the mamillary type in which the circulating blood constitutes the central compartment and there is at least one peripheral compartment, the adipose tissue. In this simple model we have two compartments, and the appropriate equations (assuming the size of the compartments remains constant) are:

$$
\begin{aligned}
& \mathrm{d} Q_{1} / \mathrm{d} t=\dot{q}-k_{12} Q_{1}+k_{21} Q_{2}-k_{\mathrm{el}} Q_{1} \\
& \mathrm{~d} Q_{2} / \mathrm{d} t=k_{12} Q_{1}-k_{21} Q_{2}
\end{aligned}
$$

where $Q_{1}$ is the quantity of the compound in the central compartment; $Q_{2}$ is the quantity in the peripheral compartment; $\dot{q}$ is the daily rate of intake into the central compartment; $k_{i j}$ are the rate constants for transfer from the $i$ th to the $j$ th compartment; and $k_{e 1}$ is the rate constant for elimination from the central compartment.

By integration we have:

$$
\begin{aligned}
& Q_{1}=A \exp \left(-m_{1} t\right)+B \exp \left(-m_{2} t\right) \\
& Q_{2}=C \exp \left(-m_{1} t\right)+D \exp \left(-m_{2} t\right)
\end{aligned}
$$

where

$$
\begin{aligned}
& A=\frac{c_{0}\left(k_{\mathrm{el}}-m_{2}\right)}{m_{1}-m_{2}}, B=\frac{c_{0}\left(m_{1}-k_{\mathrm{el}}\right)}{m_{1}-m_{2}} \\
& C=\frac{c_{0} k_{12}}{k_{21}} \times \frac{m_{2}}{m_{2}-m_{1}}, D=\frac{c_{0} k_{12}}{k_{21}} \times \frac{m_{1}}{m_{1}-m_{2}}
\end{aligned}
$$

$m_{1}$ and $m_{2}$ being functions of $k_{12}, k_{21}$ and $k_{\mathrm{el}}$, and $c_{0}$ the concentration of the compound in the central compartment at time zero. The transfer constant $\left(k_{\mathrm{el}}\right)$ for elimination from the central compartment consists of two compo- 
nents, one corresponding to the rate of elimination of the unchanged compound $\left(k_{10}\right)$, the other to the rate of elimination of the compound by metabolism $\left(k_{m}\right)$ within the central compartment. From observations with dieldrin in rats and man $k_{10}$ appears to be small compared with $k_{\mathrm{m}}$, i.e. $k_{\mathrm{el}}=k_{\mathrm{m}}$.

A useful concept (which is used below) is that of 'turnover time', the amount of substance turned over (eliminated unchanged or converted to metabolites) in a compartment per unit of time; in a simple exponential decay model (equivalent to a single-compartment model), the turnover time is that required for the concentration to decline to $1 / \mathrm{e}$ of its original value. In the case of a single-compartment model in a steady state, the turnover time is the time required for the process to metabolize an amount of the substance equal to that present in the compartment.

The trophic level model for an organochlorine compound in an ecosystem is as follows (for ease of comparison with the model given by Harrison et al. their symbolism has been used). The flow of a pesticide into the ith trophic level, and out of that level either unchanged or as metabolites, is given by:

$$
\sum_{j=1}^{i-1} c_{j} \dot{m}_{j, i}=m_{i} \frac{\mathrm{d} c_{i}}{\mathrm{~d} t}+c_{i} \dot{m}_{i, i+1}+\dot{m}_{\mathrm{nat}, i}+c_{\mathrm{ex}, i} \dot{m}_{\mathrm{ex}, i}+\dot{p}_{\mathrm{met}, i}
$$

where $c_{i}$ and $c_{j}$ are the mean concentrations of the pesticide in the $i$ th and $j$ th levels, respectively; $\dot{m}_{j, i}$ is the mean rate of transfer of mass (of the organism) from the $j$ th to the $i$ th level (thus, $c_{i} \dot{m}_{j, i}=$ input per unit time of the substance into the $i$ th trophic level, and corresponds to $a$ in equation 3 and $\dot{q}$ in equation 7); $\dot{p}_{\text {met }, i}$ is the mean rate of metabolism of the compound in organisms of the $i$ th level (corresponds to $k_{\mathrm{m}} Q_{i}$ in equation 7, assuming $k_{\mathrm{m}}=k_{\mathrm{el}}$ ); $\dot{m}_{i, i+1}$ is the mean rate of loss of biomass from the $i$ th level to the $(i+)$ th level (i.e. as a result of predation); $\dot{m}_{\text {nat }, i}$ is the mean rate of loss of biomass from the $i$ th level as a result of death by causes other than predation;

$$
\dot{m}_{i, i+1}+\dot{m}_{\mathrm{nat}, i}=\dot{m}_{\mathrm{d}, i}
$$

(as defined by Harrison et al., $\dot{m}_{\mathrm{d}, i}$ being the mean rate of loss of biomass as a result of death by all causes); $c_{\mathrm{ex}, i}$ is the mean concentration of the pesticide in the excreta of organisms of the $i$ th level (Harrison et al. use $c_{\mathrm{ex}}$, but this assumes that the concentration is the same in the excreta of organisms of all trophic levels); and $\dot{m}_{\mathrm{ex}, i}$ is the mean rate of loss of excreta from the $i$ th level. In terms of the symbolism used in equation (7):

$$
\dot{p}_{\mathrm{met}, i}+c_{\mathrm{ex}, i} \dot{m}_{\mathrm{ex}, i}=k_{10} Q_{1}
$$

There are a number of implicit assumptions in the above formulation of the kinetics of a pesticide in trophic levels. For example, the mean concentration of the pesticide in the $i$ th level is:

$$
\sum^{N_{i}} x_{i \alpha} / m_{i \alpha}
$$

where $x_{i \alpha}$ is the amount of the pesticide in all members of the $\alpha$-species, and $N_{i}$ is the number of species in the $i$ th level. The rates of loss of the various species from the $i$ th level by predation may vary quite widely, and thus the 


\section{J. ROBINSON}

rate of transfer to the $i$ th level may not be a simple function as assumed above. For the purpose of this particular model this complication is ignored since it does not invalidate the model.

If $T_{i}$ is the average time for the death of one generation of members of all species in the $i$ th trophic level, then the rate of loss of biomass from the $i$ th level is given by:

$$
\dot{m}_{\mathrm{d}, i}=m_{i} / T_{i}
$$

From equations 11-13 we have:

$$
\frac{\mathrm{d} c_{i}}{\mathrm{~d} t}+\frac{c_{i}}{T_{i}}=\sum_{j=1}^{i-1} c_{j} \dot{m}_{j, c} / m_{i}-\frac{\dot{p}_{\mathrm{met}, i}}{m_{i}}-\frac{c_{\mathrm{ex}, i} \dot{m}_{\mathrm{ex}, i}}{m_{i}}
$$

The solution in equation 14 is achieved by making the following simplifying assumptions:

(1) $\sum_{j=1}^{i-1} c_{j} \dot{m}_{j, i}$ is constant (this assumption is implicit in equation 15 of the model of Harrison et al.).

(2) The mean rate of metabolism in the ith level is directly proportional to the mean concentration in that level, i.e.

$$
\dot{p}_{\mathrm{met}, i}=k_{\mathrm{m}, i} c_{i} m_{i}
$$

where $k_{\mathrm{m}, i}$ is the mean rate constant for metabolism of the pesticide in members of the $i$ th trophic level. This is an over-simplification, but it does not affect the formal development of the model.

On the other hand, it is more realistic than the assumption of Harrison et al. that $\dot{p}_{\text {met }, i}=0$; this assumption is invalid if members of any species in the $i$ th level can metabolize the pesticide.

(3) The mean concentration of the unchanged pesticide in the excreta is directly proportional to the mean concentration in members of the $i$ th level:

$$
\frac{c_{\mathrm{ex}, i} \dot{m}_{\mathrm{ex}, i}}{m_{i}}=k_{\mathrm{ex}, i} c_{i}
$$

where $k_{\mathrm{ex}, i}$ is the mean rate constant for the excretion of the pesticide from members of the $i$ th level.

Using these assumptions we have:

$$
\frac{\mathrm{d} c_{i}}{\mathrm{~d} t}+\frac{c_{i}}{T_{i}}=\sum_{j=1}^{c-1} c_{j} \dot{m}_{j, i} / m_{i}-k_{\mathrm{ex}, i} c_{i}-k_{\mathrm{m}, i} c_{i}
$$

The solution, assuming $c_{i}=0$ when $t=0$, is:

$$
c_{i}=\frac{\sum_{j=1}^{i-1} c_{j} \dot{m}_{j, i}}{m_{i} \gamma_{i}}\left\{1-\exp \left(-\gamma_{i} t\right)\right\}
$$

where $\gamma_{i}=\left(1 / T_{i}-k_{\mathrm{ex}, i}-k_{\mathrm{m}, i}\right)$.

Thus, the rate of accumulation of the pesticide in the ith level will be greater as $\gamma_{i}$ increases, and as $t$ increases the concentration will approach the steady state value of: 


$$
\frac{\sum_{j=1}^{i-1} c_{j} m_{i, i}}{m_{i} \gamma_{i}}
$$

Harrison et al. advert to the abiotic components of the biosphere (atmosphere, hydrosphere and pedosphere), but do not incorporate the components into their model. These are important reservoirs or compartments and mathematical models of the behaviour of organochlorine compounds in the biosphere that do not incorporate these components may be quite misleading. We may simplify the total biosphere model by considering just two components, the abiotic components and the biota. The estimated dimensions of these components, and the quantities stored in them, are given in Table 1.

Table 1. Estimates of organochlorine compounds in various components of the biosphere.

\begin{tabular}{lcccc}
\hline Components of biosphere & $\begin{array}{c}\text { Mass of } \\
\text { component } \\
\text { (metric tons) }\end{array}$ & $\begin{array}{c}\text { Estimates (order of magnitude) } \\
\text { Range of mean } \\
\text { contration } \\
(\mathrm{mg} / \mathrm{kg})\end{array}$ & $\begin{array}{c}\text { Range of mean } \\
\text { chem. in } \\
\text { component } \\
\text { (metric tons) }\end{array}$ & $\begin{array}{c}\text { Turnover }^{a} \\
\text { (metric tons } \\
\text { per year) }\end{array}$ \\
\hline
\end{tabular}

\begin{tabular}{|c|c|c|c|c|}
\hline \multicolumn{5}{|l|}{ Abiotic } \\
\hline Troposphere & $10^{12}$ & $10^{-6}-10^{-5}$ & $10^{\circ}-10^{1}$ & - \\
\hline $\begin{array}{l}\text { Hydrosphere (upper } \\
\text { mixed layer, depth } 100 \mathrm{~m} \text { ) }\end{array}$ & $10^{16}$ & $10^{-6}-10^{-5}$ & $10^{4}-10^{5}$ & - \\
\hline depth $(0.6 \mathrm{~cm})$ & $10^{12}$ & $10^{-4}-10^{-3}$ & $10^{2}-10^{3}$ & - \\
\hline \multicolumn{5}{|l|}{ Biota } \\
\hline Man & $10^{8}$ & $10^{-1}-10^{0}$ & $10^{1}-10^{2}$ & $10^{1}-10^{2}$ \\
\hline Livestock & $10^{9}$ & $10^{-1}-10^{0}$ & $10^{2}-10^{3}$ & $10^{2}-10^{3}$ \\
\hline Feral animals & $10^{7}$ & $10^{-1}-10^{0}$ & $10^{0}-10^{1}$ & $2-20$ \\
\hline Terrestrial plants & $10^{12}$ & $10^{-3}-10^{-2}$ & $10^{3}-10^{4}$ & $10^{2}-10^{3}$ \\
\hline Aquatic plants & $10^{9}$ & $10^{-3}-10^{-2}$ & $10^{0}-10^{1}$ & $10^{-1}-10^{0}$ \\
\hline $\begin{array}{l}\text { Fish and other aquatic } \\
\text { animals }\end{array}$ & $10^{9}$ & $10^{-1}-10^{0}$ & $10^{2}-10^{3}$ & $10^{2}-10^{3}$ \\
\hline
\end{tabular}

${ }^{a}$ Size of central (metabolizing) compartments: man, livestock, feral animals, fish and aquatic animals, $80 \% ;$ plants, $10 \%$. Turnover times: man, $0.66 \mathrm{yr}$; livestock, $0.5 \mathrm{yr}$; feral animals, $0.25 \mathrm{yr}$; fish, $0.33 \mathrm{yr}$; plants, $1 \mathrm{yr}$.

The total size of the abiotic compartment exceeds that of the biotic compartment by several orders of magnitude, and is therefore a potentially significant storage compartment for compounds such as DDT or dieldrin. The concentrations of organochlorine compounds in the abiotic components are very small, of the order of $0.1-20 \mathrm{ng}$ per $\mathrm{kg}$ in the air; $1-200 \mathrm{ng} / \mathrm{l}$ in the hydrosphere; and $0.0003-200 \mathrm{mg} / \mathrm{kg}$ in the soil. The orders of magnitude of the mean concentrations in Table 1 are tentative but are considered to be realistic. It is apparent that the amount stored in the abiotic compartments (assuming complete mixing in the three components) is about 10 times that in the biota. However, the biotic components may not be negligible, since metabolism is occurring in at least some of the organisms. If one assumes 
that the central (metabolizing) compartments and turnover times have the values given in Table 1, then the amounts processed per year may be calculated; these estimates are also given. It is stressed that all the estimates given in Table 1 are tentative and can only be regarded as indicative of orders of magnitude and not of accurate values.

The estimates of the amounts stored in the various components, and of the annual amounts degraded, are not consistent with the output of either DDT or of aldrin and dieldrin during the past 20 years or so. Thus, the total production of DDT, 1950-1970, was probably of the order of $2 \times 10^{6}$ metric tons, of which only about 20 per cent can be accounted for in terms of the estimated quantities stored or processed in the biosphere.

There are other possible mechanisms of loss that have not been considered so far. Thus, soil organisms may have a relatively large biomass and, if they have a metabolizing ability, they may constitute a route of loss. The other mechanisms involve either chemical reactions in the atmosphere or transfer across the tropopause into the stratosphere and degradation in that part of the atmosphere. The information on the fate of quasi-inert chemicals in the atmosphere gives some indication on mechanisms of loss of pesticides in the troposphere.

\section{REMOVAL PROCESSES FOR QUASI-INERT CHEMICALS}

Some of the geochemical data relating to quasi-inert chemicals are given in Table 2.

Table 2. Geochemical data on quasi-inert chemicals.

\begin{tabular}{|c|c|c|c|}
\hline & $\begin{array}{c}\text { Estimated annual } \\
\text { emission (tons) } \\
\text { Anthropogenic Natural }\end{array}$ & $\begin{array}{l}\text { Average concentration } \\
\text { in atmosphere } \\
\text { (p.p.m., v/v) }\end{array}$ & $\begin{array}{l}\text { Residence time in } \\
\text { atmosphere } \\
\text { (year) }\end{array}$ \\
\hline $\mathrm{CO}$ (refs. 12,13 ) & $2.7 \times 10^{8} \quad 7.5 \times 10^{7}$ & 0.1 & $<3$ \\
\hline $\mathrm{CH}_{4}$ (ref. 11) & $\leftarrow \quad 1.5 \times 10^{8} \quad \rightarrow$ & 1.5 & 3 (ref. 11), 16 (ref. 14) \\
\hline $\mathrm{N}_{2} \mathrm{O}$ (ref. 15) & $-\quad 5.9 \times 10^{8}$ & 0.25 & 4 \\
\hline $\mathrm{CCl}_{3} \mathrm{~F}$ & $4.4 \times 10^{5} \quad-$ & $5 \times 10^{-5}$ & $>10$ \\
\hline
\end{tabular}

Chemical reactions with $\mathrm{O}_{2}$, atomic oxygen, ozone or nitrogen dioxide are not considered to be significant routes of removal of carbon monoxide from the atmosphere ${ }^{12,16}$; there is no significant washout or rainout of carbon monoxide; and, further, it does not partition into the oceans to any significant extent ${ }^{12}$. Consequently, to explain the present apparent steady state of the concentration of carbon dioxide, Robinson and Robbins ${ }^{12}$ have postulated that soil bacteria are removing it, or that it is absorbed in plant tissue during reduration, whereas Seiler and Junge ${ }^{13}$ have suggested that oxidation of carbon monoxide occurs in the stratosphere. This latter suggestion requires that about $3.5 \times 10^{8}$ tons of carbon monoxide are being transferred annually across the tropopause. It has also been suggested that there is sufficient energy in electrical discharges in thunderstorms to facilitate oxidation of carbon monoxide. Methane, like carbon monoxide, appears to be quite inert in the atmosphere; transport into the stratosphere is not considered an important 
route of loss ${ }^{17}$, and it has been suggested ${ }^{12}$ that methane is oxidized rapidly on the surfaces of vegetation. Nitrous oxide is partially removed by transfer across the tropopause and subsequent photodissociation in the stratosphere ${ }^{15}$, and Bates and Hayes ${ }^{18}$ have suggested that it is absorbed by plants during the photosynthetic process.

The compound trichlorofluoromethane has been studied by Lovelock and his co-workers ${ }^{19}$. They concluded that the agreement between the observed values and those predicted on the basis of an ideal inert gas was quite satisfactory. The only route of loss of this quasi-inert gas is by transfer across the tropopause and degradation in the stratosphere; this mode of loss was not discussed by Lovelock et al. and its importance cannot be assessed at present.

Three of these compounds are of interest in that they are partially or wholly of natural origin, appear to have achieved steady concentrations, and the mechanisms of removal that balance the input into the atmosphere are still speculations; however, the various suggestions, such as oxidation during thunderstorms, transfer into the stratosphere and subsequent degradation, conversion by soil bacteria, or absorption by plants during the photosynthetic process, give valuable leads in the study of the fate of other relatively inert compounds, such as the polychlorinated biphenyls and other organochlorine compounds, in the atmosphere.

Two of the processes mentioned in the speculation on the processes that may participate in the removal of quasi-inert chemicals from the atmosphere involve the enormous area of contact between the atmosphere and the earth's surface or the plant cover. The uptake of gases by vegetation or other objects on the earth's surface is conveniently expressed in terms of the deposition velocity $\left(V_{\mathbf{g}}\right)$ :

$$
\begin{gathered}
\text { deposition velocity }=\frac{\text { rate of deposition per unit area }}{\text { concentration of chemical in the }} \\
\text { atmosphere at the earth's surface }
\end{gathered}
$$

It corresponds to the transfer constant for the passage of a compound from the air to some component of the earth's surface, the compound partitioning between the air and that component. The investigations of the transport of iodine from atmosphere to grass in the field ${ }^{20}$, and the uptake of radioactive thorium by various surfaces, including grass, in wind-tunnel experiments ${ }^{21}$ give valuable insights into this aspect of the transfer of chemicals from the atmosphere. Atkins and Eggleton ${ }^{22}$ determined the partition coefficients, water/air, for lindane, dieldrin and $p p^{\prime}$-DDT, and concluded that there is reasonable agreement between the experimental partition coefficients and the wash-out ratios calculated from the published data on lindane and dieldrin; the wash-out ratio for $p p^{\prime}$-DDT was higher than expected. The deposition velocities for these three compounds on grass were also determined. These experiments imply that as regards the troposphere, pedosphere, upper mixed layer of the hydrosphere, and the vegetation cover of the earth, the concentrations of a chemical in these components should achieve a steady state in which the chemical potential of the compound is the same in these components, although soil and foliage to which pesticides are directly applied will 
function as sources and the chemical potential of a pesticide in these sub-sets of the pedosphere and biota may be greater than that in the other components for several years.

The partition coefficients for $p p^{\prime}$-DDT and dieldrin in particular could be used to predict the relative steady state concentration in air, water and vegetation, and this may provide some information on whether the environmental samples analysed to date are biased and unrepresentative of the biosphere as a whole.

\section{ALTERNATIVE FORMULATIONS OF COMPARTMENTAL MODELS}

Two other approaches, using different symbols, to the modelling of the behaviour of chemical in the biosphere, have been proposed; namely the world models of Meadows and his co-workers ${ }^{23}$, 24 , based on the methods developed by Forrester ${ }^{1}$, and the chemical analogues of Odum's energy circuit models ${ }^{25}$. It appears that the 'languages' of these two models are consistent with each other ${ }^{26}$, and with the standard mathematical symbolism used in this paper.

\section{STATUS OF MATHEMATICAL MODELS OF PESTICIDES}

The models discussed above have been based on general principles derived from studies of other chemicals, which can be plausibly extrapolated to pesticides in general, and organochlorine compounds in particular. There are obvious serious deficiencies in these models at present, which are probably at least the consequence of a lack of sufficient empirical data derived from properly designed methods of sampling. It is not possible at present to ascertain whether the proposed models, with all their simplifications, are adequate to account for the kinetics of organochlorine compounds in the biosphere, or whether a completely conceptual scheme, which differs significantly from the compartmental theory, is required.

\section{REFERENCES}

1 J. W. Forrester, Industrial Dynamics, MIT Press: Cambridge, Mass. (1961).

2 R. Harré, The Principles of Scientific Thinking, Macmillan: London (1970).

${ }^{3}$ R. Revelle and H. E. Suess, Tellus, 9, 18 (1957).

${ }^{4}$ B. Bolin and E. Eriksson, in B. Bolin (Ed.), The Atmosphere and the Sea in Motion, p 130. Rockefeller Institute Press: New York (1959).

5 C. D. Keeling, in S. I. Rasool (Ed.), Chemistry of the Lower Atmosphere, p 251. Plenum Press: New York (1973).

6 J. Robinson, Nature, 215, 33 (1967).

${ }^{7}$ H. L. Harrison, O. L. Loucks, J. W. Mitchell, D. F. Parkhurst, C. R. Tracy, D. G. Watts and V. J. Yannacone, Jr. Science, 170, 503 (1970).

8 J. Robinson, in C. A. Edwards (Ed.), Environmental Pollution by Pesticides, p 459. Plenum Press: London (1973).

9 K. R. Popper, The Logic of Scientific Discovery, Hutchinson: London (1959).

${ }^{10}$ K. R. Popper, Conjectures and Refutations, Routledge and Kegan Paul: London (1963).

11 K. R. Popper, Objective Knowledge, Clarendon Press: Oxford (1972).

12 E. Robinson and R. Robbins, Final Report, Project PR-6755, Stanford Research Institute (1968); and Supplementary Report (1969). 


\section{MATHEMATICAL MODELS IN ECOCHEMISTRY}

13 W. Seiler and C. E. Junge, J. Geophys. Res. 75, 2217 (1970).

14 T. Koyama, J. Geophys. Res. 68, 3971 (1963).

15 K. Schütz, C. E. Junge, R. Beck and B. Albrecht, J. Geophys. Res. 75, 2230 (1970).

16 P. A. Leighton, Photochemistry of Air Pollution, Academic Press; New York (1961).

17 A. E. Bainbridge and L. E. Heidr, Tellus, 18, 221 (1966).

18 D. R. Bates and̆ P. B. Hayes, Planetary Space Sci. 15, 189 (1967).

19 J. E. Lovelock, R. J. Maggs and R. J. Wade, Nature, 241, 194 (1973).

20 A. C. Chamberlain and R. Chadwick, Tellus, 18, 226 (1966).

21 A. C. Chamberlain, Proc. Roy. Soc. (London), A290, 236 (1966).

22 D. H. F. Atkins and A. E. J. Eggleton, Proc. Symp. Nuclear Techniques in Environmental Pollution, I.A.E.A., Vienna, p 521 (1971).

${ }^{23}$ J. Randers and D. L. Meadows, 'Systems simulation to test environmental policy: A sample study of DDT movement in the environment', Massachusetts Institute of Technology (1971).

24 J. Randers, in D. L. and D. H. Meadows (Eds.), Toward Global Equilibrium, Wright-Allen Press: Cambridge, Mass. (1973).

${ }^{25}$ H. T. Odum, Environment, Power and Society, Wiley/Interscience: New York (1971).

${ }^{26}$ H. T. Odum, in D. Dyrssen and D. Jaguer (Eds.), The Changing Chemistry of the Oceans, p 223. Wiley/Interscience: New York (1972). 